\title{
Optic nerve oxygen tension: effects of intraocular pressure and dorzolamide
}

\author{
M la Cour, J F Kiilgaard, T Eysteinsson, A K Wiencke, K Bang, J Dollerup, P K Jensen, \\ E Stefánsson
}

\begin{abstract}
Aim-To investigate the influence of acute changes in intraocular pressure on the oxygen tension in the vicinity of the optic nerve head under control conditions and after intravenous administration of $500 \mathrm{mg}$ of the carbonic anhydrase inhibitor dorzolamide.

Methods-Domestic pigs were used as experimental animals. Oxygen tension was measured by means of a polarographic electrode in the vitreous $0.5 \mathrm{~mm}$ anterior to the optic disc. This entity is called the optic nerve oxygen tension. Intraocular pressure was controlled by a hypodermic needle inserted into the anterior chamber and connected to a saline reservoir.

Results-When the intraocular pressure was clamped at $20 \mathrm{~cm} \mathrm{H} \mathrm{H}_{2} \mathrm{O}$ optic nerve oxygen tension was $20(5) \mathrm{mm} \mathrm{Hg}(\mathrm{n}=8)$. Intravenous administration of dorzolamide caused an increase in optic nerve oxygen tension of $43(8) \%(n=6)$. Both before and after administration of dorzolamide optic nerve oxygen tension was unaffected by changes in intraocular pressure, as long as this pressure remained below $60 \mathrm{~cm} \mathrm{H}_{2} \mathrm{O}$. At intraocular pressures of $60 \mathrm{~cm} \mathrm{H} \mathrm{H}_{2} \mathrm{O}$ and below, dorzolamide significantly increased optic nerve oxygen tension.

Conclusion-Intravenous administration of $500 \mathrm{mg}$ dorzolamide increases the oxygen tension at the optic nerve head during acute increases in intraocular pressure. (Br f Ophthalmol 2000;84:1045-1049)
\end{abstract}

University of Iceland, Reykjavik, Iceland

T Eysteinsson

E Stefánsson

Merck Sharp \&

Dohme, Glostrup,

Denmark

K Bang

J Dollerup

Correspondence to:

Dr M la Cour, Eye

Department 2061,

Rigshospitalet, Blegdamsve

9, 2100 Copenhagen $\varnothing$,

Denmark

m.la.cour@dadlnet.dk

Accepted 28 April 2000 pressure (IOP), damage to the optic nerve head, and corresponding visual field defects. Currently, elevated IOP is considered one of many risk factors for glaucomatous optic nerve damage. Increased IOP may directly injure the optic nerve fibres, or it may compromise vascular supply and thereby indrectly damage the optic nerve. ${ }^{12}$ There is overwhelming evidence that, in healthy experimental animals, blood flow in the optic nerve head is autoregulated and is therefore largely unaffected by acute increases in IOP up to $50 \mathrm{~mm} \mathrm{Hg}$ or more. ${ }^{3-9}$ In humans non-invasive measurements with the Heidelberg flow meter have shown that blood flow in the optic nerve head seems to be autoregulated to a significantly lesser degree than the inner retinal circulation. ${ }^{10}$ Recently, Evans and coworkers, ${ }^{11}$ using colour Doppler flowmetry, showed that posture induced autoregulatory changes in central retinal artery blood flow were deficient in patients with glaucoma. Autoregulation of the optic nerve head also seems to be impaired in old arteriosclerotic monkeys. ${ }^{12}$ On the other hand, in monkeys with experimental glaucoma caused by a prolonged increase in IOP, autoregulation of optic nerve head blood flow has been found to be unimpaired by acute increases in the IOP up to $75 \mathrm{~mm} \mathrm{Hg.}{ }^{6}$ This has led some to doubt whether impaired vascular supply has a role in the pathogenesis of glaucoma. ${ }^{6}$

Carbonic anhydrase inhibitors are potent vasodilators in the central nervous system. ${ }^{13}$ The use of carbonic anhydrase inhibitors before carotid artery surgery may be useful as a diagnostic test to determine the degree to which the cerebral vasculature can dilate. ${ }^{14}$ In the eye, carbonic anhydrase inhibitors seem to dilate vessels in the optic nerve head. ${ }^{15}$ We have recently shown that the optic nerve oxygen tension increases after intravenous injection of the carbonic anhydrase inhibitors acetazolamide and dorzolamide. ${ }^{16}$

Carbonic anhydrase inhibitors are widely used as antiglaucoma drugs. It is important to know whether these drugs affect autoregulation of optic nerve oxygen tension. In the present study we investigated the dependence of optic nerve oxygen tension on IOP under control conditions and after administration of the carbonic anhydrase inhibitor dorzolamide.

Part of this work has already been presented as an abstract. ${ }^{17} 18$

\section{Materials and methods}

ANAESTHESIA AND ANIMAL PREPARATION Danish domestic pigs (Danish Landrace), 25-33 kg in weight, were used as experimental animals. Their treatment was supervised by a veterinarian nurse and followed the ARVO resolution for the use of animals in ophthalmic and vision research. Permission for the use of pigs in this study was granted by the Danish board for surveillance of the use of experimen- 

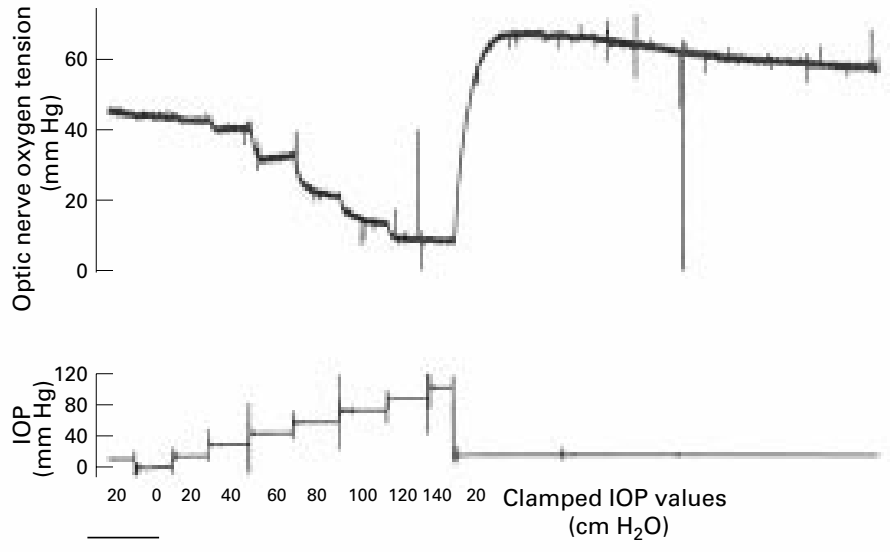

10 minutes

Figure 1 Optic nerve oxygen tension measured with a polarographic oxygen electrode placed in the vitreous approximately $0.5 \mathrm{~mm}$ anterior to the optic nerve head. The intraocular pressure (IOP) was clamped by means of a hypodermic needle inserted into the anterior chamber and connected to a saline reservoir. IOP was continuously monitored; clamped values of IOP are shown in $\mathrm{cm}_{2} \mathrm{O}$ and the actual measured values of IOP in $m m \mathrm{Hg}$.

tal animals (Danish Animal Experiments Inspectorate).

Two different methods of anaesthesia were used. Six out of a total of eight pigs were anaesthetised with midazolam (Dormicum, Roche) and ketamine (Ketalar, Parke-Davis) followed by intravenous administration of $250 \mathrm{mg}$ mebumal (Mebumal, SAD, Denmark). The anaesthesia was supplemented with fentanyl and mebumal as needed. These pigs were muscle relaxed with pancuronium (Pavlon). Two pigs were anaesthetised without the use of muscle relaxants. These pigs were given an induction dose of $200 \mathrm{mg}$ mebumal followed by an infusion of $500 \mathrm{ml}$ of a $0.4 \%$ alpha-chloralose solution. Alpha-chloralose was obtained from Fluka (Germany). Dorzolamide was obtained from Merck, Sharp \& Dohme, Glostrup, Denmark and was dissolved as a $3 \%$ solution in $100 \mathrm{mM}$ citrate buffer, $\mathrm{pH}$ 5.6 .

During anaesthesia the pigs were endotracheally intubated and artificially ventilated. The stroke volume and respiratory frequency were kept constant during the experiment. The inspired gas could be changed between different gas mixtures. The right pupil was dilated with $1 \%$ tropicamide eye drops (Mydriacyl, Alcon) and $2.5 \%$ phenylephrine eye drops (Metaoxedrin, SAD, Denmark). A catheter was placed in the femoral artery for continuous measurement of arterial blood pressure. The ECG and rectal temperature were monitored and recorded. Frequent arterial blood samples were drawn and analysed for oxygen and carbon dioxide tensions and $\mathrm{pH}$. Analysis of blood gases was performed on an ABL 605 blood gas analyser (Radiometer, Copenhagen, Denmark).

The head of the pig was secured to the operating table by surgical tape and a speculum was placed between the lids of the right eye. Two 4-0 silk traction sutures were placed in the sclera to immobilise the eye.
Intraocular pressure was controlled by adjusting the height of a saline reservoir connected to a 27 gauge hypodermic needle inserted into the anterior chamber.

\section{OXYGEN MEASUREMENTS}

Optic nerve oxygen tension was measured with a polarographic oxygen electrode that consists of a $100 \mu \mathrm{mr}$ platinum-iridium electrode inside a 20 gauge needle (Model 761, Diamond Electrotech Inc, Ann Arbor, MI, USA). The polarographic electrode was advanced through a sclerostomy placed approximately $2.0 \mathrm{~mm}$ behind the limbus. Once in the vitreous cavity, the electrode was mounted in a micromanipulator and visualised by indirect ophthalmoscopy. The tip of the polarographic electrode was placed $0.5 \mathrm{~mm}$ above the optic nerve head in an area devoid of major vessels. A silversilver chloride $(\mathrm{Ag} / \mathrm{AgCl})$ reference electrode was placed subcutaneously in the lower lid. The polarographic oxygen electrode was calibrated before each experiment in a calibrating cell containing $155 \mathrm{mM}$ saline bubbled with $100 \% \mathrm{~N}_{2}, 5 \% \mathrm{O}_{2} / 95 \% \mathrm{~N}_{2}$, and $20 \% \mathrm{O}_{2} / 80 \% \mathrm{~N}_{2}$ at $37^{\circ} \mathrm{C}$ and used as described by Stefánsson et $a l .{ }^{19}$ The oxygen tension recordings were adjusted for barometric pressure and expressed as $\mathrm{mm} \mathrm{Hg}$.

The experiments were all run with room lights on (white fluorescent light tubes). The ambient light intensity at the level of the pig's eye was found to be approximately 500 lux.

EXPERIMENTAL PROTOCOL

Seven out of eight experiments were begun with the animal breathing $100 \%$ oxygen. Once a stable optic nerve oxygen tension was obtained, the breathing mixture was changed to ambient air containing $21 \%$ oxygen. In one experiment the pig breathed air throughout the experiment. While the pig was ventilated with ambient air, and after stabilisation of optic nerve oxygen tension, the IOP was clamped at $20 \mathrm{~cm} \mathrm{H} \mathrm{H}_{2} \mathrm{O}$ and subsequently changed in steps of $20 \mathrm{~cm} \mathrm{H}_{2} \mathrm{O}$ between 0 and $140 \mathrm{~cm} \mathrm{H}_{2} \mathrm{O}$. In most experiments the sequence $20,0,20,40$, $60,80,100$, and $120 \mathrm{~cm} \mathrm{H}_{2} \mathrm{O}$ (or 15, 0, 15, 29, $44,59,74$, and $88 \mathrm{~mm} \mathrm{Hg}$ ) was tested. The IOP was kept constant until optic nerve oxygen tension had stabilised, in most cases for 3-5 minutes. Values of optic nerve oxygen tension were sampled after stabilisation-that is, during the last minutes of the applied test IOP. In six out of eight experiments the effect of dorzolamide was tested. After the initial sequence of changes in IOP, the IOP was changed back to $20 \mathrm{~cm} \mathrm{H}_{2} \mathrm{O}$ and $500 \mathrm{mg}$ dorzolamide $(16.7 \mathrm{ml}$ of a $3 \%$ solution) was administered in the femoral vein. After stabilisation of optic nerve oxygen tension, IOP was again changed as already described and the resulting changes in optic nerve oxygen tension were recorded.

DATA ANALYSIS

Individual values of IOP were tested between one and seven times in each pig. There was a considerable variation in the values of optic nerve oxygen tension between pigs. In order to 
Table 1 Mean (SE) values of arterial blood $p H$, arterial carbon dioxide and oxygen tensions $\left(\mathrm{PCO}_{2}, \mathrm{PO}_{2}\right)$ and mean arterial blood pressure before and after administration of $500 \mathrm{mg}$ dorzolamide intravenously

\begin{tabular}{|c|c|c|c|c|}
\hline & Before dorzolamide & After dorzolamide & $n$ & $p$ value \\
\hline Arterial blood pH (units) & $7.64(0.05)$ & $7.49(0.04)$ & 6 & $<0.0005$ \\
\hline Arterial blood $\mathrm{PCO}_{2}(\mathrm{kP})$ & $3.4(0.4)$ & $5.1(0.5)$ & 6 & $<0.0005$ \\
\hline Arterial blood $\mathrm{PO}_{2}(\mathrm{kP})$ & $13 \quad(1)$ & 13 & 6 & 0.3 \\
\hline Mean arterial blood pressure $(\mathrm{mm} \mathrm{Hg})$ & (7) & 84 & 6 & 0.09 \\
\hline
\end{tabular}

The number of pigs (n), and the $\mathrm{p}$ value for the paired $t$ test are indicated.

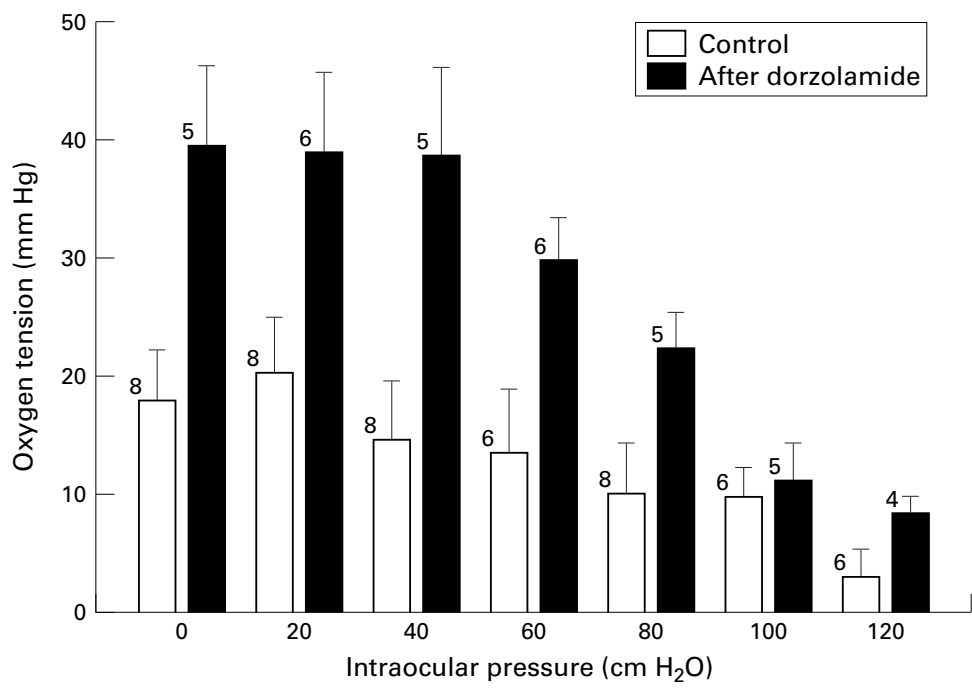

Figure 2 Optic nerve oxygen tension measured at different values of intraocular pressure in the domestic pig. Measurements were performed before and after administration of $500 \mathrm{mg}$ dorzolamide intravenously. Error bars indicate standard errors; the number of pigs is indicated at each bar.

avoid skewness in the analysis, we calculated the mean value of optic nerve oxygen tension for each value of IOP in each pig before and after administration of dorzolamide. The analysis was conducted from these means.

\section{Results}

In seven pigs the animal was initially ventilated with pure oxygen. When the inspired gas was changed from pure oxygen to air (21\% oxygen), the optic nerve oxygen tension decreased from a mean value of 41 (SE 7) $\mathrm{mm} \mathrm{Hg}$ to 21 (4) $\mathrm{mm} \mathrm{Hg}$. This is similar to our previously reported finding. ${ }^{16}$

Figure 1 shows a typical experiment where a sequence of increasing IOPs was investigated. It is seen that optic nerve oxygen tension remained relatively constant for IOP up to $40 \mathrm{~cm} \mathrm{H} \mathrm{H}_{2} \mathrm{O}$. Only after the IOP was increased to $80 \mathrm{~cm} \mathrm{H}_{2} \mathrm{O}$ did optic nerve oxygen tension drop precipitously. Above $120 \mathrm{~cm} \mathrm{H}_{2} \mathrm{O}$ further increases in IOP did not influence optic nerve oxygen tension. The mean arterial blood pressure was around $70 \mathrm{~mm} \mathrm{Hg}$ during this experiment. Note the large overshoot in optic nerve oxygen tension after the IOP returned from $140 \mathrm{~cm} \mathrm{H}_{2} \mathrm{O}$ to $20 \mathrm{~cm} \mathrm{H}_{2} \mathrm{O}$. After challenge with high values of IOP we kept the IOP at $20 \mathrm{~cm} \mathrm{H}_{2} \mathrm{O}$ until optic nerve oxygen tension had stabilised (15-60 minutes). The optic nerve oxygen tension observed after runoff of these overshoots was similar to that measured in the same pig at the beginning of the experiments, before the IOP had been changed. The difference was $5(7) \mathrm{mm} \mathrm{Hg}(n=8)$ which is not statistically significantly different from zero (paired $t$ test, $\mathrm{p}>0.1$ ).

In six pigs the effects of dorzolamide were studied. The results of intravenous administration of $500 \mathrm{mg}$ dorzolamide on arterial $\mathrm{pH}$, arterial oxygen and carbon dioxide tensions, and mean arterial blood pressure are shown in Table 1. Dorzolamide caused an increase in arterial carbon dioxide tension and a corresponding decrease in arterial $\mathrm{pH}$, but no changes in mean arterial blood pressure or in arterial oxygen tension. These changes are similar to our previously reported findings. ${ }^{16}$

Administration of dorzolamide was performed while the IOP was clamped at $20 \mathrm{~cm} \mathrm{H} \mathrm{H}_{2} \mathrm{O}$. After administration of dorzolamide the optic nerve oxygen tension increased by a mean of $43($ SE 8$) \%(n=6)$. This is similar to our previously reported finding in experiments where the IOP was not clamped. ${ }^{16}$

When we compared the six experiments where the animals were relaxed and anaesthetised with mebumal and fentanyl and the two experiments where the animals were not relaxed and were anaesthetised with alphachloralose we found no differences in the effects of dorzolamide and the relation between IOP and optic nerve oxygen tension. Thus, the described findings seem to be independent of the anaesthesia used.

Figure 2 summarises the results of the experiments similar to that shown in Figure 1, performed before and after administration of dorzolamide. The optic nerve oxygen tension was largely unaffected by changes in IOP as long as this was below $60 \mathrm{~cm} \mathrm{H}_{2} \mathrm{O}$ (Fig 2). This was the case both before and after administration of dorzolamide. Figure 2 includes all data in the study, including our first two pigs in whom dorzolamide was not administered. For unknown reasons these two pigs had smaller optic nerve oxygen tensions than the subsequent six pigs, all of which received the drug. In the two pigs not given dorzolamide the mean optic nerve oxygen tension measured at an IOP of $20 \mathrm{~cm} \mathrm{H}_{2} \mathrm{O}$ was 14 (10) $\mathrm{mm} \mathrm{Hg}$. In the remaining six pigs which did receive the drug the optic nerve oxygen tension was 22 (6) $\mathrm{mm} \mathrm{Hg}$. Figure 2 shows the influence of the IOP on optic nerve oxygen tension under control conditions and after administration of dorzolamide. However, at any given value of IOP, Figure 2 overestimates the effects of the drug on optic nerve oxygen tension.

To obtain a true assessment of the effects of dorzolamide on the optic nerve oxygen tension we compared the optic nerve oxygen tensions measured before and after administration of the drug in the same pig. Figure 3 shows these paired differences in optic nerve oxygen tension for each of the investigated values of IOP. Dorzolamide significantly increased optic nerve oxygen tension for IOP values of $60 \mathrm{~cm} \mathrm{H}_{2} \mathrm{O}$ and below.

\section{Discussion}

We have previously shown that it is possible to make reliable measurements of the oxygen tension in the normal pig vitreous using the relatively large polarographic oxygen electrode 


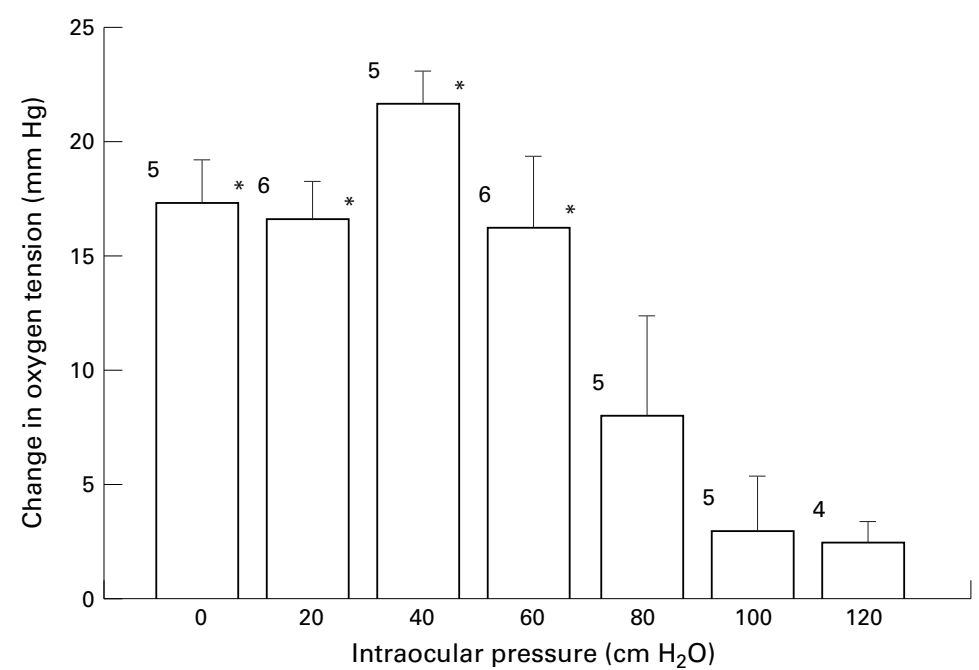

Figure 3 Difference in optic nerve oxygen tension between the control state and after administration of $500 \mathrm{mg}$ dorzolamide intravenously. Error bars indicate standard errors; the number of pigs where paired measurements were made are indicated at each bar. ${ }^{\star} p<0.05$ (paired, one sided t tests).

used in the present study. ${ }^{16}$ The vitreous acts as a diffusion medium, with very low endogenous oxygen consumption. The oxygen gradients within the vitreous are small (about $2 \mathrm{~mm} \mathrm{Hg}$ per mm distance). ${ }^{20}$ The oxygen tension in vitreous $0.5-1.0 \mathrm{~mm}$ in front of the optic nerve is therefore relatively insensitive to the exact distance of the sensor to the optic nerve head, and we interpret the measured oxygen tension as reflecting the average oxygen tension in the superficial part of the optic nerve head, and denote it optic nerve oxygen tension.

Earlier measurements with microelectrodes have shown that the oxygen tension in front of the optic nerve head is unevenly distributed. ${ }^{5}{ }^{21}$ In a study in minipigs the oxygen tension in the vitreous overlying optic nerve head arterioles was $33 \mathrm{~mm} \mathrm{Hg}$ during normoxia and increased to $81 \mathrm{~mm} \mathrm{Hg}$ during hyperoxia $(100 \%$ oxygen). In vitreous overlying the optic nerve head, but not adjacent to arterioles, the oxygen tension was $17 \mathrm{~mm} \mathrm{Hg}$ and remained unchanged during hyperoxia. ${ }^{21}$ Our measurements probably represent a spatial average of optic nerve head oxygen tensions, although some contribution from the adjacent retina cannot be excluded. We carefully placed the electrode over an area without major vessels. Accordingly, we found oxygen tensions between previously reported values for vitreal oxygen tension measured adjacent to optic nerve head arterioles, and oxygen tension measured away from such arterioles. Both during normoxia and hyperoxia, our values were closest to previously reported oxygen tensions over optic nerve head tissue away from major vessels. $^{21}$ In our experiments the oxygen tension in arterial blood remained unchanged after intravenous administration of dorzolamide (Table 1). It is therefore not likely that the changes in optic nerve oxygen tension observed after administration of this drug were solely caused by altered diffusion of oxygen out of arterioles.

The measured changes in optic nerve oxygen tension could be caused by changes in oxygen delivery or changes in oxygen consumption (metabolism). We assume that changes in oxygen delivery were responsible for the major part of the observed changes in optic nerve oxygen tension. We have previously found that the optic nerve oxygen tension increases as expected when the optic nerve head blood vessels are dilated by the well known CNS vasodilators acetazolamide and carbon dioxide. ${ }^{16} \mathrm{We}$ have also reported that the changes in optic nerve oxygen tension closely reflect changes in blood flow in the optic nerve head, as measured by a laser Doppler flow probe. ${ }^{22}$

The present experiments show that the optic nerve oxygen tension in the pig is autoregulated. This autoregulation is most obvious when the vessels have been dilated by the prior administration of dorzolamide (Figs 1 and 2). Only at IOP above $60 \mathrm{~cm} \mathrm{H}_{2} \mathrm{O}$ did we observe a precipitous drop in the optic nerve oxygen tension. An IOP of $60 \mathrm{~cm} \mathrm{H}_{2} \mathrm{O}$ corresponds to an intraocular perfusion pressure of approximately $35 \mathrm{~mm} \mathrm{Hg}$ if this entity is defined as the mean arterial blood pressure minus the IOP. With the IOP increased to $120 \mathrm{~cm} \mathrm{H}_{2} \mathrm{O}$ the intraocular perfusion pressure was around $-7 \mathrm{~mm} \mathrm{Hg}$. With this IOP we found optic nerve oxygen tensions not significantly different from zero under control conditions. After administration of dorzolamide there might have been some pulsatile flow during the systole, since positive optic nerve oxygen tensions were measured (Fig 2). The present findings are in agreement with previous studies of autoregulation of blood flow in the optic nerve head. ${ }^{3-9}$

We have previously shown that intravenous administration of the carbonic anhydrase inhibitors acetazolamide and the more potent dorzolamide increase the optic nerve oxygen tension in a dose dependent manner. ${ }^{16}$ The present experiments indicate that carbonic anhydrase inhibition does not inhibit autoregulation of the optic nerve oxygen tension. Instead, after carbonic anhydrase inhibition this autoregulation is preserved, but the level of optic nerve oxygen tension is shifted upwards (Fig 2).

At all values of IOP tested the optic nerve oxygen tension was higher after administration of dorzolamide than in controls (Fig 3). The present experiments confirm earlier preliminary findings that the effects of dorzolamide on the optic nerve oxygen tension is a direct effect of the drug on the optic nerve head circulation, rather than an indirect effect due to a lowering of the IOP. ${ }^{16}$

Dorzolamide was administered only after a series of raised IOPs had been tested in the absence of the drug. The advantage of this approach is that each pig can serve as its own control in assessing the effects of the drug. However, it may be argued that local vasodilators are released during the period of high IOP and that their effects are present during the remaining course of the experiment. This would cause an overestimation of the vasodilating effect of dorzolamide. We cannot rule out this possibility. However, after a series of raised IOPs had been tested the optic nerve oxygen 
tension stabilised at values that were not statistically significantly different from those found at the beginning of the experiments before the IOP was altered.

The vasculature of the optic nerve head in the pig has many similarities to that in humans but it is more clearly trilaminar with a more well defined arterial circle of Zinn-Haller. ${ }^{23}$ If the findings of the present investigation can be generalised to the human optic nerve head circulation, intravenous dorzolamide, and perhaps other carbonic anhydrase inhibitors too, may be useful in preserving oxygenation of the optic nerve head during acute episodes of increased IOP in otherwise healthy young eyes. However, it must be remembered that these experiments were carried out in healthy young pigs and have only a theoretical bearing on the situation in patients with glaucoma in whom the optic nerve head circulation and its regulation may have been damaged by the disease.

The assistance of Mette Olesen and veterinary nurse Letty Klarskov and other staff at the Experimental Surgery Unit,
Panum Institute, University of Copenhagen is greatly appreciPanum Institute, University of Copenhagen is greatly appreci\& Dohme.

1 Flammer J. The vascular concept of glaucoma. Surv Ophthalmol 1994;38(Suppl):S3-6.

2 Hayreh SS. Inter-individual variation in blood supply of the optic nerve head. Its importance in various ischemic disorders of the optic nerve head, and glaucoma, low-tension glaucoma and allied disorders. Doc Ophthalmol 1985;59: $217-46$.

3 Ahmed J, Linsenmeier RA, Dunn RJ. The oxygen distribution in the prelaminar optic nerve head of the cat. Exp Eye Res 1994;59:457-65.

4 Alder VA, Cringle SJ. Intraretinal and preretinal $\mathrm{PO}$ response to acutely raised intraocular pressure in cats. $A m$ f Physiol 1989;256:H1627-34

5 Pournaras CJ, Munoz JL, Abdesselem R. [Regulation of $\mathrm{PO}_{2}$ in the area of the optic papilla of the minipig in hyperoxia]. Klin Monatsbl Augenheilkd 1991;198: minipig

6 Quigley HA, Hohman RM, Sanchez R, et al. Optic nerve head blood flow in chronic experimental glaucoma. Arch Ophthalmol 1985;103:956-62.
7 Riva CE, Cranstoun SD, Petrig BL. Effect of decreased ocular perfusion pressure on blood flow and the flickerinduced flow response in the cat optic nerve head. Microvasc Res 1996;52:258-69.

8 Shonat RD, Wilson DF, Riva CE, et al. Effect of acute increases in intraocular pressure on intravascular optic nerve head oxygen tension in cats. Invest Ophthalmol Vis Sci 1992;33:3174-80.

9 Sperber GO, Bill A. Blood flow and glucose consumption in the optic nerve, retina and brain: effects of high intraocular pressure. Exp Eye Res 1985;41:639-53.

10 Michelson G, Groh MJ, Langhans M. Perfusion of the juxtapapillary retina and optic nerve head in acute ocular hypertension. Ger f Ophthalmol 1996;5:315-21.

11 Evans DW, Harris A, Garrett M, et al. Glaucoma patients demonstrate faulty autoregulation of ocular blood flow during posture change. Br f Ophthalmol 1999;83:809-13.

12 Hayreh SS, Bill A, Sperber GO. Effects of high intraocular pressure on the glucose metabolism in the retina and optic nerve in old atherosclerotic monkeys. Graefes Arch Clin Exp Ophthalmol 1994;232:745-52.

13 Vorstrup S, Henriksen L, Paulson OB. Effect of acetazolamide on cerebral blood flow and cerebral metabolic rate for oxygen. $\mathcal{F}$ Clin Invest 1984;74:1634-9.

14 Okudaira Y, Bandoh K, Arai H, et al. Evaluation of the acetazolamide test. Vasoreactivity and cerebral blood volume. Stroke 1995;26:1234-9.

15 Eysteinsson T, Bang K, la Cour M, et al. The effect of carbonic anhydrase inhibition on optic nerve tension in the pig. IOVS (ARVO Supplement) 1998;39:s693 (Abstract).

16 Stefansson E, Jensen PK, Eysteinsson T, et al. Optic nerve oxygen tension in pigs and the effect of carbonic anhydrase inhibitors. Invest Ophthalmol Vis Sci 1999;40:2756-61.

17 La Cour M, Eysteinsson T, Bang K, et al. Optic nerve tension in the pig. IOVS (ARVO Supplement) 1998;39:s694 (Abstract).

$18 \mathrm{La}$ Cour M, Eysteinsson T, Kiilgaard JF, et al. Optic nerve oxygen tension. The effect of intraocular pressure and carbonic anhydrase inhibition. IOVS (ARVO Supplement) 1999;40:s491 (Abstract)

19 Stefánsson E, Hatchell DL, Fisher BL, et al. Panretinal photocoagulation and retinal oxygenation in normal and diabetic cats. Am f Ophthalmol 1986;101:657-64.

$20 \mathrm{~A} \operatorname{lm} \mathrm{A}$, Bill A. The oxygen supply to the retina. I. Effects of changes in intraocular and arterial blood pressures, and in arteria $\mathrm{PO}_{2}$ and $\mathrm{PCO}_{2}$ on the oxygen tension in the vitreous body of the cat. Acta Physiol Scand 1972;84:261-74.

21 Bouzas EA, Donati G, Pournaras CJ. Distribution and regulation of the optic nerve head tissue $\mathrm{PO}_{2}$. Surv Ophthalmol 1997;42(Suppl 1):S27-34

22 Jensen PK, Eysteinsson $\mathrm{T}$, Bang $\mathrm{K}$, et al. Effect of dorzolamide on the blood flow in the optic nerve head of normal pigs. In: Pillunat LE, Harris A, Anderson DR, eds.Current concepts on ocular blood flow in glaucoma. The Hague, Netherlands: Kugler Publications, 1999:267-71.

23 Rootman J. Vascular system of the optic nerve head and retina in the pig. Brf Ophthalmol 1971;55:808-19. 